\title{
Comparing Circadian Rhythms in Blood Pressure Computed From the Arterial Line and Sphygmomanometer in the ICU
}

\author{
Eoin Finnegan ${ }^{1}$, Mauricio Villarroel ${ }^{1}$, Shaun Davidson ${ }^{1}$, Mirae Harford $^{1,2}$, Joao Jorge ${ }^{1}$, Lionel \\ Tarassenko $^{1}$ \\ ${ }^{1}$ Institute of Biomedical Engineering, Department of Engineering Science, University of Oxford \\ ${ }^{2}$ Critical Care Research Group, Nuffield Department of Clinical Neurosciences, University of Oxford
}

\begin{abstract}
It has previously been shown that there is an underlying bias in blood pressure (BP) measurements between the arterial line and the sphygmomanometer cuffs. It is unclear the extent to which this bias can lead to differences in the standard of care inside the Intensive Care Unit (ICU). The objective of this study was to compare the circadian patterns of systolic, mean arterial and diastolic $B P$ (SBP, MAP and DBP) derived from simultaneous measurements of arterial lines and sphygmomanometer cuffs in the ICU. 2,243 patients were selected from the MIMIC-III database, resulting in 22,743 data-points of paired BP measurements from the arterial line and sphygmomanometer cuff. A significant bias between the two measurement sources was found for SBP and MAP but not for DBP. In addition, a significant proportion (SBP - 51\%, DBP - 23\%, MAP - 41\%) of cuff measurements had a difference greater than $10 \mathrm{mmHg}$ with respect to the arterial line. Despite these errors, the circadian rhythms between the two signals showed a high correlation: SBP - $r=0.92 p<0.001 ;$ MAP $-r=0.90 p<0.001 ; D B P$ - $r=0.86 p<0.001$. Despite significant errors and bias between the arterial line and cuff, the sphygmomanometer cuff is sufficiently accurate to track circadian rhythms in $B P$ in the ICU.
\end{abstract}

\section{Introduction}

The arterial line is generally considered to be the gold standard of blood pressure (BP) measurement in the intensive care unit (ICU) [1]. However, arterial lines are invasive and can lead to complications such as occlusion of the vessel, bleeding from the insertion site and infection [1]. Additionally, the arterial line can be impacted by under or over dampening of the catheter, which can affect the estimates of systolic and diastolic blood pressure (SBP and DBP respectively). Alternatively, non-invasive blood pressure can be intermittently measured using a sphygmomanometer cuff and the oscillometric technique.
Measurements of blood pressure using sphygmomanometer cuffs have known limitations depending on: posture, movement, cuff-inflation hypertension and cuff size [2].

In the ICU, a patient may have their blood pressure monitored simultaneously by both a sphygmomanometer cuff and an arterial line for numerous reasons. These include: checking for under or over dampening of the arterial line; during a transition period where a patient is moving from arterial line to cuff (or vice versa); or for double monitoring of blood pressure. Previous studies $([3,4])$ have highlighted a bias in measurements of blood pressure between the two techniques. However, the extent to which this bias can affect the evaluation of circadian rhythms in blood pressure is unknown.

There is a growing emphasis in the ICU to support a patient's natural circadian rhythms [5]. The natural circadian rhythm in blood pressure is well documented and deviations from it can contain significant prognostic information [6]. To the authors' knowledge there has been no work which compares the circadian rhythms in blood pressure computed from the arterial line and sphygmomanometer cuff in the ICU.

\section{Methods}

\subsection{Dataset}

Data for this study was drawn from the Medical Information Mart for Intensive Care III (MIMIC III) [7] database. MIMIC contains critical care information gathered between 2001 and 2012 from the Beth Israel Deaconess Medical Centre (BIDMC) in Boston, Massachusetts, USA between 2001 and 2012. We selected periods during which a patient had both the arterial line waveform and cuff measurements recorded.

\subsection{Blood pressure estimation from the arterial line}

The arterial blood pressure (ABP) signal recorded in the MIMIC database was sampled at $125 \mathrm{~Hz}$. The 
bedside monitor used throughout the study also provided estimates of SBP, MAP and DBP sampled at either $1 \mathrm{~Hz}$ or $1 / 60 \mathrm{~Hz}$, depending on the software version used at the time of recording. The algorithms for estimating BP were undisclosed by the manufacturer. In addition, an assessment of the quality of the blood pressure (BP) estimates is not included in the database. Signal quality can reveal periods during which the ABP signal was affected by motion artefacts, under dampening, over dampening or noise. We propose that re-estimating these metrics from the ABP signal, together with an assessment of their signal quality and combining these with the time series produced by the bedside monitor, would provide more reliable and robust blood pressure estimates.

Systolic and diastolic blood pressure are typically defined as the peak and trough of the arterial blood pressure signal respectively. We followed the work of Villarroel et al. [8] for detecting the peaks and onsets of a pulsatile signal. In order to suppress high-frequency noise whilst preserving the pusatile component, the ABP signal was first filtered using a low-pass 10th-order Butterworth filter with a cut-off frequency of $16 \mathrm{~Hz}$. MAP was estimated for each beat as [9] MAP $=\frac{2}{3} \mathrm{DBP}+\frac{1}{3} \mathrm{SBP}$

\subsection{Signal quality index of the arterial line}

A signal quality index (SQI) was computed for each beat of the ABP signal as a number varying between 0 and 1 , indicating periods of poor or good signal quality respectively. An accurate estimate of blood pressure could then be determined from periods of good-quality signal. Villarroel et al. [8] proposed an SQI for pulsatile signals. The SQI detected time periods during which the signal was clipped, or sharp changes in amplitude occurred, or heart rate was outside of physiological bounds or beats that did not match a running-average windowed template. In addition, Sun et al. [10] proposed a rule-based signal abnormality index for ABP waveforms. The combined SQI of the ABP for each beat of the ABP signal was determined by multiplying the SQIs from Villarroel et al. and Sun et al. For an ABP signal, these algorithms were able to locate periods of motion artefacts, incorrectly attached lines, errors in the beat detection algorithm and sudden changes in beat morphology.

To provide a robust estimate of BP from the arterial line, the recomputed beat-by-beat BP values, using the methods outlined above, were fused with the time series of SBP, MAP and DBP estimated by the bedside monitor from the ABP signal. Periods for which there was a disparity of more than $10 \mathrm{mmHg}$ between the recomputed time series and the estimates from the bedside monitor were flagged as poor quality and the SQI was set as 0 . This threshold was set by AAMI, ESH and ISO as a tolerable error for a blood pressure measuring device [11]. The reference blood pressure signal for use in this work was determined as the average of the recomputed BP values and the bedside monitor estimates at each time step.

\subsection{Comparing blood pressure values from the arterial line with cuff readings}

To compare the time series of BP estimated from the arterial line with the spot measurements from the sphygmomanometer cuff, the arterial line signals were averaged over a 10 minute window, centred on the times of cuff inflation. Only data of good quality (deemed to have an SQI > 0.8) were used in the average. If less than $60 \%$ of the window was deemed to be good quality, then a valid estimate of BP from the arterial line was not calculated.

Errors in readings of the BP cuff can be expected in recordings from a hospital dataset. Cuff readings are often verified by nurses prior to being put into the clinical information system. However, due to the busy nature of the ICU, errors in this verification process are inevitable [4]. In addition, no information regarding patient movement at the time of cuff inflation was provided, which can have a significant impact on the readings obtained. We considered a valid data-point to include a value for SBP, MAP and DBP from both the arterial line and the cuff. Outliers from this distribution were located using Hadi's method [12]. The underlying distribution was approximated and any data points that were found to deviate significantly from the centre of the distribution were said to be outliers. The distance metric used in Hadi's method was the Mahalanobis distance. As recommended in Hadi et al. [12], a threshold on the distance metric should be set by visual inspection. In this work, we chose a threshold of 6 to be a good indicator of outliers in the distribution.

In order to compare the values of blood pressure computed by the sphygmomanometer cuff and the arterial line, we performed two forms of analysis. First, we studied a global comparison of all pairs of BP from the two devices across the entire dataset. This analysis was performed to highlight any underlying biases and errors between the two methods. The global comparison of BP from the arterial line and the cuff was performed using a Bland-Altman analysis on the pairs of readings of SBP, MAP and DBP across the entire cohort.

The second analysis compared the circadian rhythm in BP computed by the two devices. We followed the work of Davidson et al [5] who showed that a circadian pattern in BP can be observed in an ICU by averaging across a large cohort of patients. The evaluation of the circadian rhythms of SBP, MAP and DBP from the arterial line and cuff was performed by averaging all measurements in each one-hour period of the day across the entire cohort. The mean hourly values were recorded left-aligned (e.g. the 

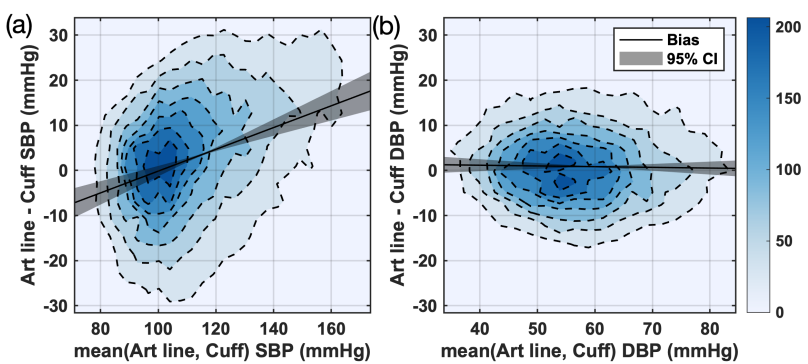

Figure 1: Global comparison of (a) SBP and (b) DBP measurements from the arterial line and sphygmomanometer cuff. The colour scale applies to all three plots.

figure

mean of the measurements between 1:00am and 1:59am was recorded as occurring at 1:00am). The circadian patterns are compared via Pearson's pairwise correlation coefficient of the 24 mean hourly values.

\section{Results}

A total of 2,243 patients across 2,344 ICU stays were selected. $57.5 \%$ of the cohort was male, $69.1 \%$ was white. 22,743 datapoints of estimated blood pressure from both the arterial line and cuff were computed.

Figure 1 shows the results of the global comparison of blood pressure computed from the arterial line and sphygmomanometer cuff. For SBP, the mean error was $3.56 \mathrm{mmHg}$, with a standard deviation of $16.7 \mathrm{mmHg}$. For MAP, the mean error was $6.77 \mathrm{mmHg}$, with a standard deviation of $10.0 \mathrm{mmHg}$. For DBP, the mean error was $0.85 \mathrm{mmHg}$, with a standard deviation of $9.91 \mathrm{mmHg}$. 53.1 $\%$ of SBP measurements from the cuff differed from the arterial line by more than $10 \mathrm{mmHg}$. For DBP the figure was $26.2 \%$ and for MAP, $39.0 \%$.

Figure 2 shows the comparison of the circadian rhythms computed from the BP estimated from the arterial line (method 1 - shown in red) and the cuff (method 2 - shown in blue). The correlation coefficients of the circadian rhythms computed by the two methods were: SBP - 0.919, MAP - 0.90, DBP - 0.863. All of the correlation coefficients had a $p$ value less than 0.001 . Table 1 shows the peak-nadir excursions for SBP, MAP and DBP from the two methods. For SBP and MAP there was a lower peak-nadir excursion when measured by a sphygmomanometer cuff, rather than the arterial line. For DBP there was a slight increase.

\section{Discussion}

All mean errors fell within the $10 \mathrm{mmHg}$ guidelines from the BP device standards authorities (AAMI, ESH and ISO) [11]. However, the guidelines also state that

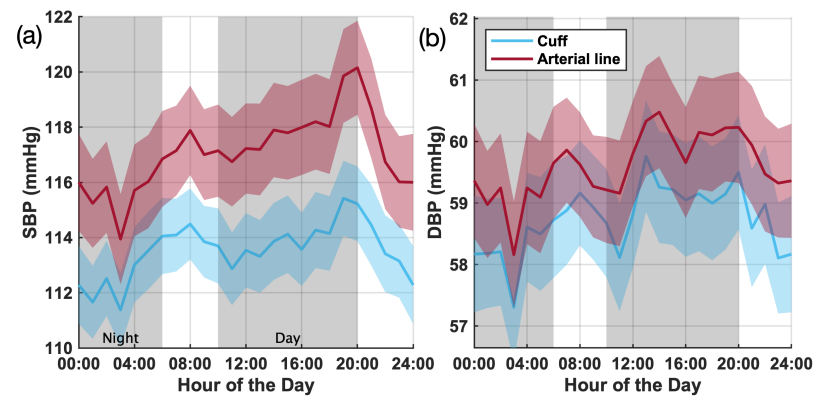

Figure 2: Comparison of circadian rhythms computed by data from both the arterial line and the cuff for (a) SBP, (b) DBP. figure

Table 1: Peak-nadir excursion results. All results are given in units of $\mathrm{mmHg}$

table

\begin{tabular}{l|ccc} 
Measurement device & SBP & MAP & DBP \\
\hline Arterial line & 6.2 & 3.4 & 2.3 \\
Sphygmomanometer cuff & 4.0 & 2.4 & 2.5 \\
\hline
\end{tabular}

$85 \%$ of all measurements must have errors lower than $10 \mathrm{mmHg}$ which we found neither SBP, MAP or DBP estimates fulfilled. We found DBP had the lowest mean error and standard deviation. SBP measurements had the largest standard deviation. This can be attributed to the fact that SBP typically has higher variability in measurements and therefore more extreme values could be expected.

A possible explanation for the large errors may be due to the set-up inside the ICU. The arterial line and the BP cuff were likely to be on opposing arms of patients. The mean errors, therefore, could be explained by the differences in BP between left and right arm which is reported to be as much as $10 \mathrm{mmHg}$ [13]. However, as arterial lines are frequently switched from arm to arm in order to avoid infection, we would suggest that the effect of the differences in BP between left and right arm will be averaged out across the dataset.

Figure 1 shows that there was an underlying bias in SBP estimates from the cuff when compared to the arterial line. Low values of BP were over-estimated and high values of $\mathrm{BP}$ were under-estimated. This followed what is reported in the literature. Interestingly, however, DBP estimates did not exhibit the same underlying bias.

All circadian rhythm correlations were high $(R>0.85)$ and statistically significant $(p<0.001)$. The profiles for SBP and MAP roughly compare visually to the typical circadian rhythm reported outside of the ICU [5], with a rise in BP upon awakening, a rise just before night time and a dip in BP overnight. The peak-nadir excursions (seen in table 1) were significantly lower than the values reported in a healthy population $(\approx 25.9 \mathrm{mmHg}[14])$. 
However they were similar to the peak-nadir excursions reported for a typical ICU population $(\approx 5 \mathrm{mmHg}[5])$. The SBP and MAP circadian rhythms measured by the sphygmomanometer cuff were slightly dampened, shown by lower peak-nadir excursion. This can most likely be explained due to the underlying bias found in SBP and MAP. The circadian rhythms in DBP from the arterial line and cuff had the most overlap caused by the lowest mean error between the two measurements.

Circadian rhythms are not typically monitored in an ICU. However, there is growing support behind this notion [5]. This work suggests that in the absence of invasive beat-by-beat values of BP, irregularly sampled, non-invasive BP measurements from the sphygmomanometer cuff could provide sufficient information for an assessment of circadian rhythms in BP.

A limitation to this work, however, was the computation of the circadian rhythms. As no patients in our cohort had both arterial line and sphygmomanometer cuff data for 24 hours, an individual comparison of circadian rhythms was not possible. Therefore, circadian rhythms were approximated by averaging across the entire cohort as is performed by Davidson et al [5]. Although the number of datapoints in each 1-hour window ranged from 853 to 1060, the median number of data-points per ICU stay was 4 and therefore the circadian rhythms should be considered as an approximation.

\section{Conclusion}

We have shown that point measurements of blood pressure using the sphygmomanometer cuff will often significantly deviate from blood pressure measurements taken by the arterial line, considered to be the gold standard. This deviation has been shown to be greater than the acceptable limits set by governing bodies. In addition, the sphygmomanometer cuff has an underlying bias in which low values of SBP or MAP are over-estimated and high values are under-estimated. However, despite these sources of errors, we have shown that computation of circadian rhythms of blood pressure from the sphygmomanometer cuff are highly correlated with circadian rhythms computed from the arterial line.

\section{References}

[1] Hager HH, Burns B. Artery Cannulation. 2019.

[2] Ogedegbe G, Agyemang C, Ravenell JE. Masked hypertension: Evidence of the need to treat. Current Hypertension Reports 2010;12(5):349-355. ISSN 15226417.

[3] Wax DB, Lin HM, Leibowitz AB. Invasive and concomitant noninvasive intraoperative blood pressure monitoring: observed differences in measurements and associated therapeutic interventions. Anesthesiology 2011; 115(5):973-978. ISSN 0003-3022.

[4] Li-wei HL, Saeed M, Talmor D, Mark R, Malhotra A. Methods of blood pressure measurement in the ICU. Critical Care Medicine 2013;41(1):34.

[5] Davidson S, Villarroel M, Harford M, Finnegan E, Jorge J, Young D, Watkinson P, Tarassenko L. Vital-sign circadian rhythms in patients prior to discharge from an ICU: a retrospective observational analysis of routinely recorded physiological data. Critical Care 2020;24(1):1-13. ISSN 1364-8535.

[6] Dolan E, Stanton A, Thijs L, Hinedi K, Atkins N, McClory S, Hond ED, McCormack P, Staessen JA, O'Brien E. Superiority of ambulatory over clinic blood pressure measurement in predicting mortality: The Dublin outcome study. Hypertension 2005;46(1):156-161. ISSN 0194911X.

[7] Johnson AE, Pollard TJ, Shen L, Lehman LWH, Feng M, Ghassemi M, Moody B, Szolovits P, Anthony Celi L, Mark RG. MIMIC-III, a freely accessible critical care database. Scientific Data 2016;3:160035.

[8] Villarroel M. Non-Contact Vital Sign Monitoring in the Clinic. Ph.D. thesis, Oxford University, 2017.

[9] Ward M, Langton JA. Blood pressure measurement. Continuing Education in Anaesthesia Critical Care and Pain 2007;7(4):122-126. ISSN 17431824.

[10] Sun JX, Reisner AT, Mark RG. A signal abnormality index for arterial blood pressure waveforms. In Computers in Cardiology, volume 33. Citeseer. ISBN 1424425328. ISSN 02766574, 2006; 13-16.

[11] O'Brien E, Petrie J, Littler W, de Swiet M, Padfield PL, O'Malley K, Jamieson M, Altman D, Bland M, Atkins N. The British Hypertension Society protocol for the evaluation of automated and semi-automated blood pressure measuring devices with special reference to ambulatory systems. Journal of Hypertension 1990; 8(7):607-619. ISSN 0263-6352.

[12] Hadi AS. Identifying multiple outliers in multivariate data. Journal of the Royal Statistical Society Series B Methodological jul 1992;54(3):761-771. ISSN 0035-9246.

[13] Clark CE, Taylor RS, Shore AC, Campbell JL. The difference in blood pressure readings between arms and survival: primary care cohort study. BMJ 2012;344:e1327. ISSN 0959-8138.

[14] Morris CJ, Hastings JA, Boyd K, Krainski F, Perhonen MA, Scheer FA, Levine BD. Day/night variability in blood pressure: Influence of posture and physical activity. American Journal of Hypertension 2013;26(6):822-828. ISSN 08957061.

Address for correspondence:

Eoin Finnegan

Old Road Campus Research Building, Roosevelt Drive, Oxford OX3 7DQ

eoin.finnegan@eng.ox.ac.uk 